\title{
Hygric properties of porous building materials (IV): semi- permeable membrane and psychrometer methods for measuring moisture storage curves
}

\author{
Chi Feng*, Hans Janssen \\ KU Leuven, Department of Civil Engineering, Building Physics Section, 3001 Leuven, Belgium
}

\begin{abstract}
The moisture storage curve is an important hygric property of porous building materials. It describes the moisture storage characteristic of a material via the relation between the moisture content and the moisture potential. Current experimental protocols for measuring moisture storage curves are not satisfactory, as they often require extensive facilities while still lacking the capability to handle the adsorption process in most of the over-hygroscopic range. This paper proposes two novel and simple methods - the semi-permeable membrane method and the psychrometer method - to solve these issues. Measurements on calcium silicate, autoclaved aerated concrete and ceramic brick are performed and validated by comparing the experimental outcomes obtained from these two novel methods and from other existing protocols. It is proven that the semi-permeable membrane method and the psychrometer method are applicable for both adsorption and desorption processes, the latter both from saturated and capillary moisture content. Limited by the accuracy of the psychrometer and the condensation caused by temperature fluctuations, these two methods show their best applicable range for capillary pressures below $-1 \cdot 10^{5} \mathrm{~Pa}$. The semi-permeable membrane method and the psychrometer method also feature in low cost, simple handling and application efficiency.
\end{abstract}

Keywords: porous building material; moisture storage curve; capillary pressure; moisture content; semi-permeable membrane; psychrometer

\section{Introduction}

Moisture is one of the central issues in buildings in particular and in the built environment in general. It affects the energy efficiency and service life of buildings [1,2], as well as the indoor climate and air quality $[3,4]$. Due to this critical nature of moisture, many hygrothermal models have been established and various numerical tools have been implemented [5-7] to optimally understand and correctly control different moisture-related processes in built structures.

For this spectrum of theoretical models and numerical tools, the hygric properties of porous building materials are always indispensable parameters. They characterize the capability of a material to store and transport moisture, and therefore can be classified as moisture storage and transport properties. In this study we concentrate on the storage properties, while in a future paper of this series we will focus on the transport properties.

Generally speaking, the moisture storage properties express the relation between the moisture content $\left(w, \mathrm{~kg} \cdot \mathrm{m}^{-3}\right)$ and the moisture potential. The moisture potential can be characterized by relative humidity $(\mathrm{RH},-)$, capillary pressure $\left(p_{c}, \mathrm{~Pa}\right)$ and other parameters, which are mutually convertible. Due to the hysteresis phenomenon, the moisture storage properties are usually not a single function 
Postprint: Feng C, Janssen H. 2019. Hygric properties of porous building materials (IV): semi-permeable membrane and psychrometer methods for measuring moisture storage curves, Building and Environment, 152: 39-49.

doi: https://doi.org/10.1016/j.buildenv.2019.01.054

but rather process-dependent scanning curves. Consequently, at least three important curves are needed for a complete characterization over the full humidity range - one adsorption curve starting from the dry state as well as two desorption curves starting from the saturated moisture content $\left(w_{s a t}, \mathrm{~kg} \cdot \mathrm{m}^{-3}\right)$ and the capillary moisture content $\left(w_{c a p}, \mathrm{~kg} \cdot \mathrm{m}^{-3}\right)$, respectively. This is illustrated in Fig.1.

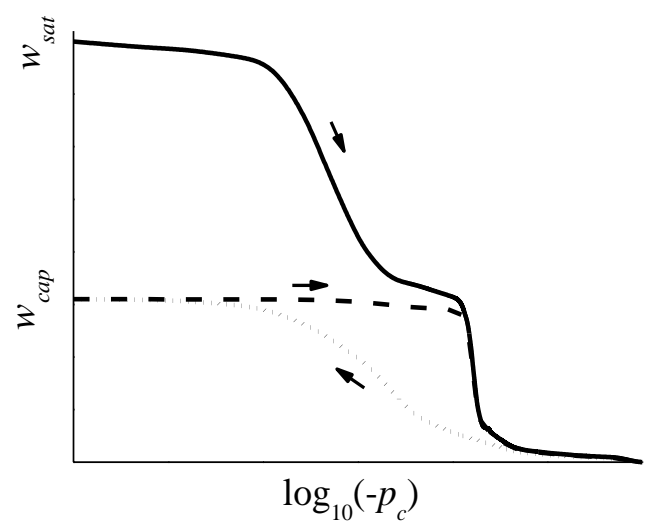

Fig.1 Moisture retention curves for different adsorption/desorption processes

Moisture storage curves can be obtained via simulation or measurement, and experiments at present form the dominant approach. Many methods have been developed in soil science and other disciplines to measure the moisture retention curves of porous materials. As is shown in Fig.2, the main distinction between the different methods is their protocols for imposing or determining the moisture potential. Consequently, these methods can be generally classified into two large categories - suction control methods and moisture control methods [8]. Suction control methods typically impose predetermined moisture potentials on the sample. When equilibrium has been reached, the moisture content of the sample can easily be obtained in a gravimetric way. Contrarily, moisture control methods typically condition the sample for its moisture content and then determine the corresponding moisture potential.

Both suction control methods and moisture control methods can be further elaborated. For suction control methods, there are three main techniques to control the moisture potential - the axis translation technique, the osmotic technique and the ambient RH control technique. The axis translation technique was first invented by Hilf in 1956 to avoid the cavitation of water [9] and later evolved into different forms, the most popular of which is the well-known pressure plate [10-13]. In the osmotic technique solutions of different concentrations - and hence different osmotic pressures - are employed to control the moisture potential $[14,15]$. Obviously, both the axis translation and the osmotic technique suit the liquid-dominant cases (in building physics terms: the over-hygroscopic range). For vapor-dominant situations (in building physics terms: the hygroscopic range), the moisture potential control reverts to regulating the ambient $\mathrm{RH}$, typically realized with saturated salt solutions $[16,17]$ or mixing dry/wet air [18]. With respect to moisture control methods, the moisture content of the sample can be imposed either directly (e.g. by applying tiny water drops on the sample or even through immersion $[8,19,20]$ ) or indirectly (e.g. by contacting moist soil [21]). The determination of the moisture potential can also be performed directly (e.g. with an RH sensor or a psychrometer [8, 22]) or indirectly (e.g. the filter paper method $[10,18,23])$. 

psychrometer methods for measuring moisture storage curves, Building and Environment, 152: 39-49.

doi: https://doi.org/10.1016/j.buildenv.2019.01.054

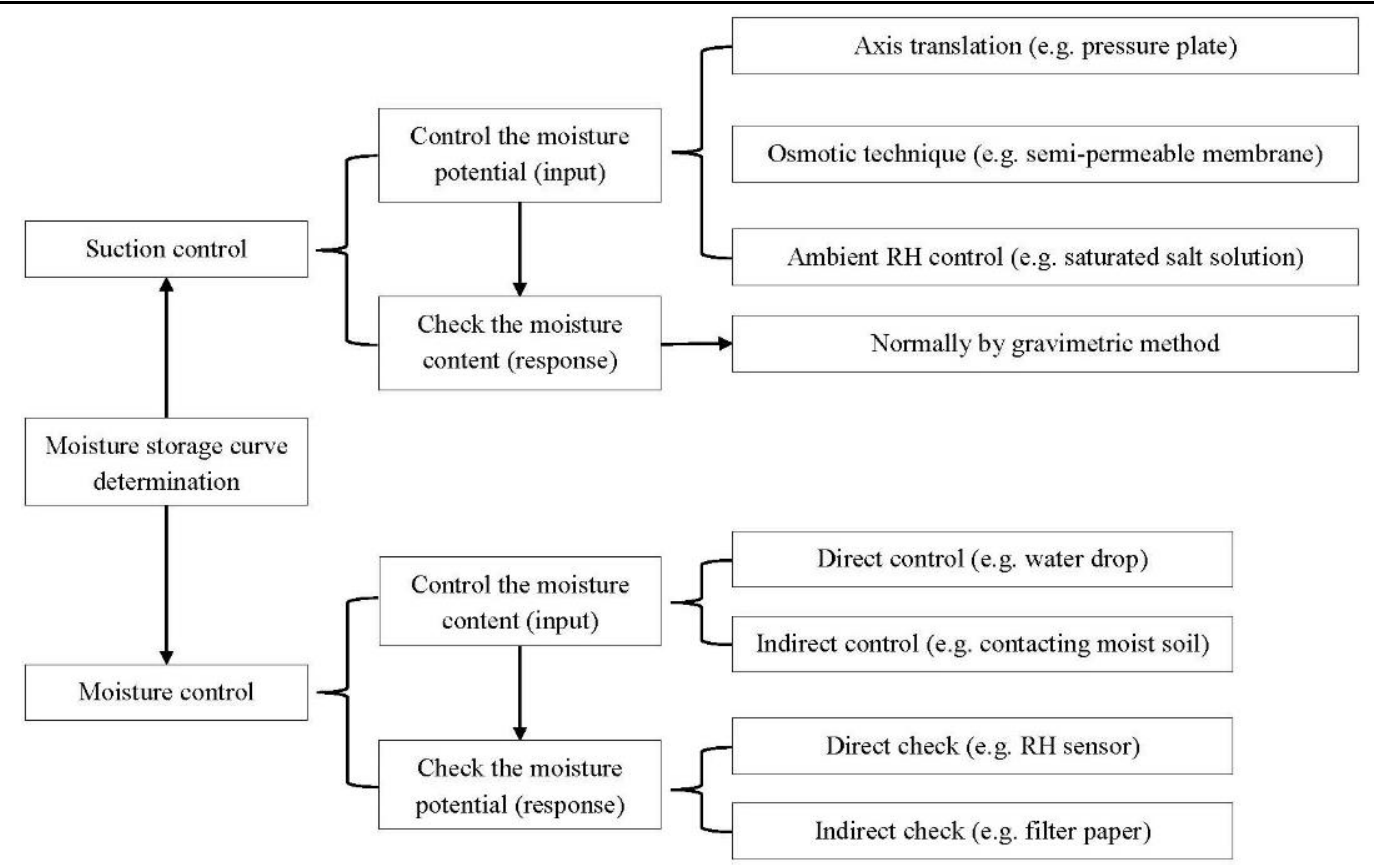

Fig.2 General methods for the determination of moisture storage curves

Unfortunately, given the differing materials' mechanical characteristics, targeted humidity ranges and adsorption/desorption processes, as well as other aspects, the above-mentioned methods are not always optimal for porous building materials. For example, the filter paper method is a standardized approach that can be applied in a wide capillary pressure range from $-1 \cdot 10^{4}$ to $-1 \cdot 10^{8} \mathrm{~Pa}$ [24]. However, it is not widely used in building physics due to the fact that most porous building materials are much more rigid than soils and the subsequent difficulty in ensuring good hydraulic contact between the sample and the filter paper. On the other hand, there are also other methods, that are not always applicable to soils but widely used for porous building materials, such as the mercury intrusion porosimetry (MIP) [25].

For building materials, the desiccator test is the most widely adopted method in the hygroscopic range. It is simple, reliable, and applicable to both adsorption and desorption processes, with either manual or automatic experimental facilities available $[16,17]$. The detailed experimental procedures can be found in the ISO 12571 standard [26] or the ASTM C1498 standard [27], as well as in many other publications. In the over-hygroscopic range on the other hand, the measurement methods for the moisture storage curves are far more demanding and far less rewarding. There are several experimental approaches available, like the (modified) pressure plate test, pressure membrane test, hanging water column test or MIP. These methods however suffer from critical limitations and are thus far from satisfactory. Table 1 summarizes their fundamental characteristics. Clearly, none of them can measure the complete moisture storage behavior in the over-hygroscopic range. Even when combined, much information for the adsorption process is still not measurable.

To solve the problems in measuring the moisture storage curves of porous building materials (especially in the over-hygroscopic range), this paper proposes two novel experimental methods - the semi-permeable membrane method and the psychrometer method. Both methods feature in simplicity, reliability and most importantly, the capability to measure both adsorption and desorption in the overhygroscopic range. In the following sections, the basic principles and procedures of these two novel methods are explained first. Next, the validation measurements on calcium silicate, autoclaved aerated 
Postprint: Feng C, Janssen H. 2019. Hygric properties of porous building materials (IV): semi-permeable membrane and psychrometer methods for measuring moisture storage curves, Building and Environment, 152: 39-49.

doi: https://doi.org/10.1016/j.buildenv.2019.01.054

concrete and ceramic brick are performed. After that experimental results from these two methods are analyzed and validated by mutual comparison, as well as by the comparison with data from other existing protocols.

Table 1 Characteristics of methods for measuring moisture retention curves of building materials

\begin{tabular}{|c|c|c|c|c|c|}
\hline Method & Pressure plate & $\begin{array}{l}\text { Pressure } \\
\text { membrane }\end{array}$ & $\begin{array}{c}\text { Modified } \\
\text { pressure plate }\end{array}$ & $\begin{array}{l}\text { Hanging water } \\
\text { column }\end{array}$ & MIP \\
\hline Standard example & ASTM C1699 [28] & ISO 11274 [29] & Unstandardized & ISO 11274 [29] & ASTM D4404 [30] \\
\hline Application example & Ref. [31] & Ref. [32] & Ref. [33] & Ref. [34] & Ref. [25] \\
\hline Equipment cost & Expensive & Expensive & Expensive & Cheap & Very expensive \\
\hline Availability & Common & Common & Rare & Rare & Common \\
\hline Toxicity & Low & Low & Low & Low & High (mercury) \\
\hline Experimental procedure & Complicated & Complicated & Complicated & Moderate & Simple \\
\hline Data processing & Simple & Simple & Simple & Simple & Complicated \\
\hline Applicable $p_{c}$ range $(\mathrm{Pa})$ & $0 \sim-1.5 \cdot 10^{6}$ & $-1.5 \cdot 10^{6} \sim-1 \cdot 10^{7}$ & $0 \sim-5 \cdot 10^{5}$ & $0 \sim-5 \cdot 10^{4}$ & $0 \sim-5 \cdot 10^{7}$ \\
\hline Applicable process & $\begin{array}{c}\text { Desorption from } \\
w_{\text {sat }} \text { and } w_{c a p}\end{array}$ & $\begin{array}{c}\text { Desorption from } \\
w_{\text {sat }} \text { and } w_{c a n}\end{array}$ & Adsorption & $\begin{array}{c}\text { Desorption from } \\
w_{\text {sat }} \text { and } w_{c a n}\end{array}$ & $\begin{array}{c}\text { Desorption from } \\
w_{\text {sat }} \text { only }\end{array}$ \\
\hline Duration & Weeks $\sim$ months & Weeks $\sim$ months & Weeks $\sim$ months & Weeks $\sim$ months & Hours $\sim$ days \\
\hline Additional remarks & $\begin{array}{c}\text { Not accurate for } \\
p_{c}>-1 \cdot 10^{5} \mathrm{~Pa}[35]\end{array}$ & & & & $\begin{array}{c}\text { Not suitable for } \\
\text { cement-based } \\
\text { materials }[36,37] \text {. }\end{array}$ \\
\hline
\end{tabular}

\section{Materials and methods}

In this section, we will first present the target materials used for validation experiments. After that, two existing protocols - the desiccator test and the pressure plate test - will be concisely introduced, as they serve as the reference methods in this study. Finally, the basic principles and procedures of the semi-permeable membrane method and the psychrometer method - which belong to the suction control methods and the moisture control methods respectively - for measuring moisture storage curves in the over-hygroscopic range will be proposed.

\subsection{Materials}

To validate the proposed semi-permeable membrane and psychrometer methods, measurements are performed on three materials - calcium silicate (CS), autoclaved aerated concrete (AAC) and ceramic brick (CB). Table 2 [38] summarizes some crucial properties of these materials: the bulk density ( $\rho_{b u l k}$, $\left.\mathrm{kg} \cdot \mathrm{m}^{-3}\right)$ and open porosity $(\phi)$ obtained from the vacuum saturation test [39], the vapor diffusion resistance factor $(\mu)$ derived from the dry cup test [40], the equilibrium moisture content at $\mathrm{RH} 84.7 \%$ $\left(u_{84.7 \%}, \mathrm{~kg} \cdot \mathrm{kg}^{-1}\right)$ determined through the desiccator test [26], the median pore-throat radius $\left(r_{50}, \mathrm{~m}\right)$ measured from the MIP test [41], as well as the capillary absorption coefficient $\left(A_{\text {cap }}, \mathrm{kg} \cdot \mathrm{m}^{-2} \mathrm{~s}^{-0.5}\right)$ and capillary moisture content $\left(w_{c a p}, \mathrm{~kg} \cdot \mathrm{m}^{-3}\right)$ tested by the capillary absorption test [38].

It is clearly revealed in Table 2 that CS is strongly hygroscopic and capillary, AAC is strongly hygroscopic but weakly capillary, and CB is weakly hygroscopic but strongly capillary. Because of these variations, these three materials are able to represent a wide variety of porous building materials with different hygric characteristics, and are therefore often chosen as target materials for hygric property characterization $[31,38,42,43]$.

For the semi-permeable membrane and psychrometer tests, raw materials are cut into samples with a diameter of $3 \mathrm{~cm}$ and a thickness of $0.5 \mathrm{~cm}$. For the desiccator and pressure plate tests, a sample size of $5 \mathrm{~cm}$ in diameter and $0.5 \mathrm{~cm}$ in thickness is chosen. The dry mass of all samples is determined by an electronic balance with a resolution of $1 \mathrm{mg}$ after drying in a ventilated oven at $70^{\circ} \mathrm{C}$, and all later wet masses are measured with the same balance. All measurements are conducted at $23 \pm 0.5^{\circ} \mathrm{C}$. 
Postprint: Feng C, Janssen H. 2019. Hygric properties of porous building materials (IV): semi-permeable membrane and psychrometer methods for measuring moisture storage curves, Building and Environment, 152: 39-49.

doi: https://doi.org/10.1016/j.buildenv.2019.01.054

\begin{tabular}{|c|c|c|c|c|c|c|c|}
\hline \multicolumn{8}{|c|}{ Table 2 Fundamental properties of target materials $\left(21-23^{\circ} \mathrm{C}\right)[38]$} \\
\hline Material & Pbulk $\left(\mathrm{kg} \cdot \mathrm{m}^{-3}\right)$ & $\phi(\%)$ & $\mu^{\mathrm{a}}$ & $u_{84.7 \%}{ }^{\mathrm{b}}\left(\mathrm{kg} \cdot \mathrm{kg}^{-1}, \%\right)$ & $r_{50}(\mathrm{~m})$ & $A_{c a p}{ }^{\mathrm{c}}\left(\mathrm{kg} \cdot \mathrm{m}^{-2} \mathrm{~s}^{-0.5}\right)$ & $w_{c a p}\left(\mathrm{~kg} \cdot \mathrm{m}^{-3}\right)$ \\
\hline $\mathrm{CS}$ & 271 & 89.1 & 2.0 & 2.9 & $3.2 \times 10^{-7}$ & 1.01 & 756 \\
\hline AAC & 462 & 81.4 & 7.4 & 3.1 & $-d$ & 0.046 & 312 \\
\hline $\mathrm{CB}$ & 1818 & 32.6 & 11.6 & $<0.05^{\mathrm{d}}$ & $4.1 \times 10^{-6}$ & 0.61 & 210 \\
\hline $\begin{array}{l}{ }^{\mathrm{a}} \text { Determi } \\
{ }^{\mathrm{b}} \text { Adsorpt } \\
{ }^{\mathrm{c}} \text { Values f } \\
{ }^{\mathrm{d}} \text { Cannot }\end{array}$ & $\begin{array}{l}\mathrm{d} \text { from dry cu } \\
\text { from dry sta } \\
20^{\circ} \mathrm{C} \text {; } \\
\text { determined a }\end{array}$ & $\begin{array}{l}\text { est (RI } \\
\text { rately. }\end{array}$ & & $-53.5 \%)$ & & & \\
\hline
\end{tabular}

\subsection{Methods}

\subsubsection{Existing methods for comparison}

To verify the semi-permeable membrane test and the psychrometer test (explained later), we perform validation measurements based on two existing methods - the desiccator test and the pressure plate test.

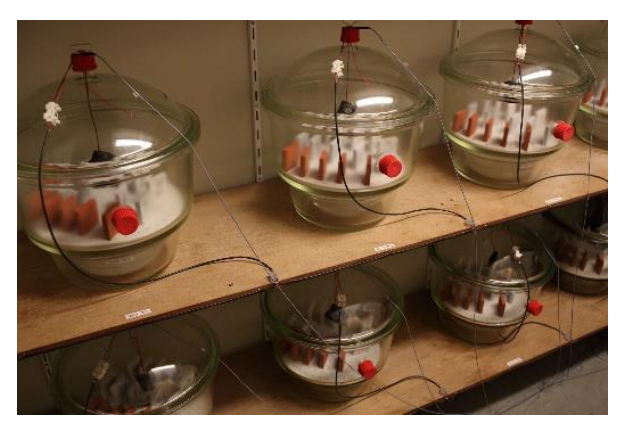

a. The desiccator test

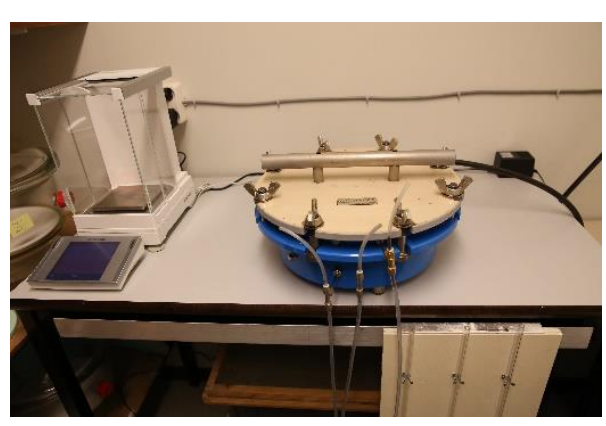

b. The pressure plate test

Fig.3 Existing experimental methods for comparison

As mentioned in Section 1, the desiccator test (Fig.3 a) is a mature and reliable method for measuring the moisture storage curves in the hygroscopic range. During the test, a constant ambient $\mathrm{RH}$ is established by a saturated salt solution (or other techniques). A correctly pre-conditioned sample is exposed to this known $\mathrm{RH}$ and its moisture content is measured gravimetrically, after reaching equilibrium. By altering the controlled $\mathrm{RH}$, the moisture content changes as well. Finally, the hygroscopic moisture storage curves can be obtained by fitting the discrete points with a proper function.

The curves obtained from the desiccator test should smoothly extend to or overlap with the lowhumidity end in the over-hygroscopic range. For this reason, we carry out the desiccator test in the $p_{c}$ range starting from $-3.6 \cdot 10^{6} \mathrm{~Pa}$ for both the adsorption from the dry state and the desorption from $w_{\text {sat }}$, based on our normal procedures [16] as well as the ISO 12571 and ASTM C1498 standards [26, 27]. We do not measure the desorption from $w_{c a p}$, because in the hygroscopic range the desorption curves from $w_{s a t}$ and $w_{c a p}$ overlap with each other for the target materials in this study, and hence are not distinguishable.

The pressure plate (Fig. $3 \mathrm{~b}$ ) test is another well-established approach, originating from soil science [44] and adopted in many other disciplines [11,32, 35, 45]. During the test, compressed air is used to exert a constant capillary pressure on the sample. When equilibrium has been reached, the moisture content with respect to that specific pressure is measured gravimetrically. By changing the exerted air 
Postprint: Feng C, Janssen H. 2019. Hygric properties of porous building materials (IV): semi-permeable membrane and psychrometer methods for measuring moisture storage curves, Building and Environment, 152: 39-49.

doi: https://doi.org/10.1016/j.buildenv.2019.01.054

pressure, different points in the moisture storage curves can be obtained.

In this study, we follow our previous experimental protocol [31, 42], which is modified based on the ISO 11274 and ASTM C1699 standards [28, 29]. A Soilmoisture ${ }^{\circledR}$ ceramic plate extractor system is used for measuring the desorption curves starting from both $w_{\text {sat }}$ and $w_{\text {cap }}$ at a $p_{c}$ range of $-1 \cdot 10^{4} \mathrm{~Pa}$ $\sim-1.5 \cdot 10^{6} \mathrm{~Pa}$.

\subsubsection{The semi-permeable membrane method}

The semi-permeable membrane method can be deemed as a derivative of the desiccator test: while saturated salt solutions are often used in the hygroscopic range for humidity control, unsaturated salt solutions can be similarly utilized for maintaining desired moisture potentials in the over-hygroscopic range. By exposing samples to such environment, the over-hygroscopic equivalent of the desiccator test can be achieved.

There are, however, two critical issues concerning the usage of unsaturated salt solutions in the overhygroscopic range. First, it is liquid, rather than vapor, that dominates the moisture transfer in the overhygroscopic range. Consequently, the surface transfer resistance of vapor diffusion will result in an enormous amount of time for the sample to reach equilibrium if directly exposed to humid air. Moreover, a slight temperature fluctuation can cause a large shift of the air humidity in the overhygroscopic range due to the limited thermal inertia and moisture capacity of air.

To solve these two problems, we keep the sample in close contact with the salt solution, with a piece of semi-permeable membrane in between to allow the moisture transport but block the salt penetration. The salt solution can also effectively buffer the fluctuations of temperature and moisture potential. When equilibrium is reached, the sample should have the same $p_{c}$ as the solution. This is the basic principle of the semi-permeable membrane method for measuring moisture storage curves (Fig.4). This method is obviously applicable to a wide moisture content range for both adsorption and desorption processes.

During the test, samples are pre-conditioned to a desired initial moisture content (dry state, $w_{\text {sat }}$ or $\left.w_{c a p}\right)$ and then laid in a sample holder whose bottom surface is a semi-permeable membrane (Fig. 5 a). The top of the sample holder is covered by a piece of plastic film to minimize the impact of condensation caused by temperature fluctuations. Next, multiple sample holders with samples inside are placed into desiccators, floating on unsaturated $\mathrm{K}_{2} \mathrm{SO}_{4}$ solutions of different concentrations (Fig.5 b). When the mass of samples no longer changes (after approximately $3 \sim 4$ weeks), the final $m_{\text {wet }}$ is recorded. Do to the moisture transfer across the semi-permeable membrane, the concentrations of $\mathrm{K}_{2} \mathrm{SO}_{4}$ solutions will slightly deviate from the original values, and their $p_{c}$ will change accordingly. We use a WP4C psychrometer (explained in the next section) to measure the final $p_{c}$.

It should be noted that ideally no salt can cross the semi-permeable membrane, while in practice a tiny amount of salt may still penetrate. The membrane used in this study is an industrial-level reverse osmosis membrane (Filmtech ${ }^{\circledR}$ Flat Sheet BW30) with a $\mathrm{NaCl}$ rejection capability as high as $99.5 \%$ [46]. The salt used in our test is $\mathrm{K}_{2} \mathrm{SO}_{4}$. Since $\mathrm{K}^{+}$is larger than $\mathrm{Na}^{+}$in size, and $\mathrm{SO}_{4}{ }^{2-}$ is larger than $\mathrm{Cl}^{-}$ in both size and charge amount, it is more difficult for $\mathrm{K}_{2} \mathrm{SO}_{4}$ to pass through the membrane. The impact from salt transport can thus be reasonably neglected.

It should be mentioned that semi-permeable membranes have previously been used for determining the moisture storage curves of porous materials $[14,15]$. However, since there is no need to study the confining stress effect on our materials, our protocol is very simple and efficient, with the capability to test multiple samples at the same time in easily-available setups. 


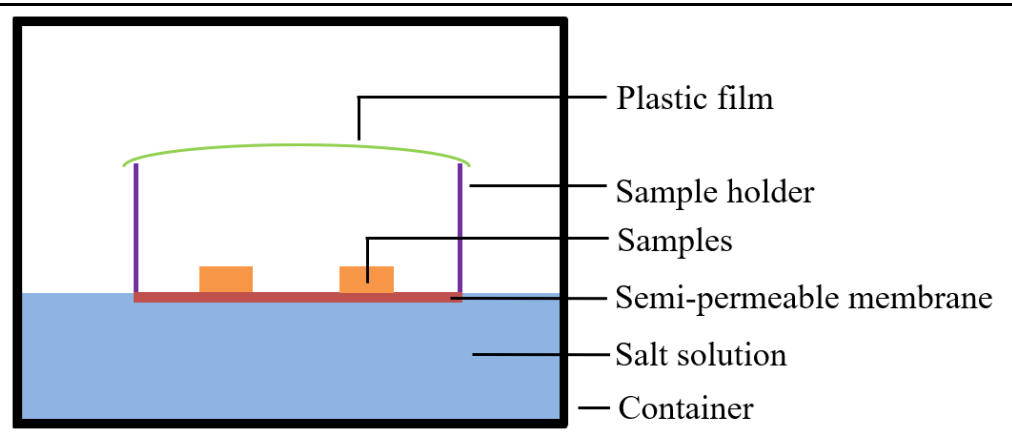

Fig.4 A schematic of the semi-permeable membrane setup

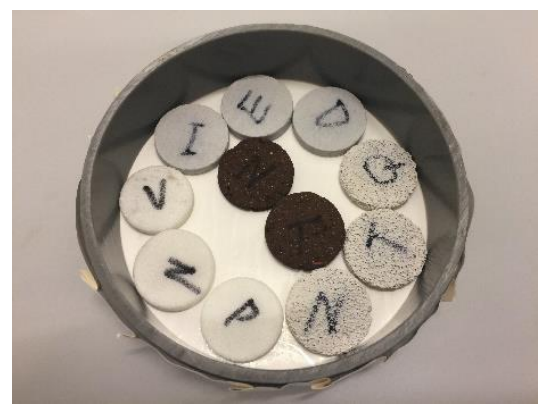

a. Samples lying on the semi-permeable membrane

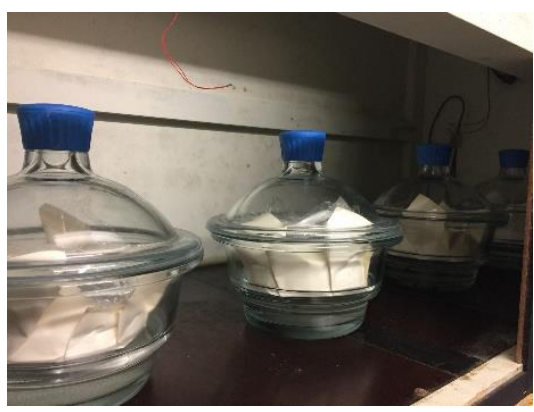

b. Samples in desiccators with salt solutions

Fig.5 The semi-permeable membrane method for measuring moisture storage curves

\subsubsection{The psychrometer method}

The psychrometer method is a suction control method. During the test, the sample is conditioned to an arbitrary moisture content first, and the $p_{c}$ in the sample is then obtained by holding the sample in a sealed chamber and measuring the humidity of the air inside caused by the water evaporation from the sample. In the over-hygroscopic range, the resultant air humidity is rather close to saturation. Consequently, the widely used RH sensors are no longer reliable because they are mainly designed for applications at a lower humidity, while psychrometers specifically designed for the high humidity range become a much better choice instead.

There are different types of psychrometers, such as the transistor psychrometer and the chilledmirror dew-point psychrometer. Many factors - such as temperature, hysteresis, calibration and equilibrium time - all have an impact on the accuracy. After comprehensive comparisons, Cardoso et al [47] recommended the chilled-mirror dew-point psychrometer. In this study, we follow their recommendation and adopt the chilled-mirror dew-point psychrometer for the humidity measurement. The instrument model is WP4C (Fig.6 a and b), produced by Decagon Devices Inc. According to the manufacturer, this psychrometer has an accuracy of $\pm 5 \cdot 10^{4} \mathrm{~Pa}$ in the range of $0 \sim-5 \cdot 10^{6} \mathrm{~Pa}$ and $\pm 1 \%$ for $-5 \cdot 10^{6} \sim-3 \cdot 10^{8} \mathrm{~Pa}$ [48]. More details about this psychrometer can be found in [49].

As a matter of fact, psychrometers have been widely used in soil science, geology and other disciplines to determine the moisture retention curves for a long time $[8,22,49]$. However, as mentioned before, the function of a psychrometer is measuring, rather than controlling the moisture potential. Consequently, the sample conditioning protocols and the adsorption/desorption processes can differ significantly. Here we propose a method which is optimal for most porous building materials.

For adsorption measurements, samples are first pre-conditioned at an ambient RH of $97 \%$ controlled by a saturated $\mathrm{K}_{2} \mathrm{SO}_{4}$ solution (Fig.7 a). Next, samples are placed above pure water in desiccators 
Postprint: Feng C, Janssen H. 2019. Hygric properties of porous building materials (IV): semi-permeable membrane and psychrometer methods for measuring moisture storage curves, Building and Environment, 152: 39-49.

doi: https://doi.org/10.1016/j.buildenv.2019.01.054

placed in an insulated chamber for better temperature stability (Fig.7 b and c). From time to time, the adsorption process is interrupted by sealing samples into small sample cups (Fig.7 d). After a standing period, the profiles of moisture content and potential in the sealed samples are assumed equilibrated. The $w$ and $p_{c}$ of samples are then measured by the balance and by the WP4C psychrometer, respectively. Resultantly, the adsorption curve starting from dry state in the over-hygroscopic range is determined. For calcium silicate and autoclaved aerated concrete, the adsorption progresses very slowly when the humidity is extremely high, while for ceramic brick the adsorption directly from air can hardly result in an observable change in moisture content. For those cases, we apply some tiny water drops directly on the samples for acceleration.

Reversibly, desorption curves starting from $w_{\text {sat }}$ or $w_{\text {cap }}$ can be obtained. For desorption measurements, the samples are first pre-conditioned to $w_{\text {sat }}$ or $w_{\text {cap }}$ and then exposed to $97 \%$ or $94 \%$ ambient $\mathrm{RH}$ (controlled by saturated $\mathrm{K}_{2} \mathrm{SO}_{4} / \mathrm{KNO}_{3}$ solutions). With a similar process, the $w$ and $p_{c}$ can be measured, and the desorption curves are hence available.

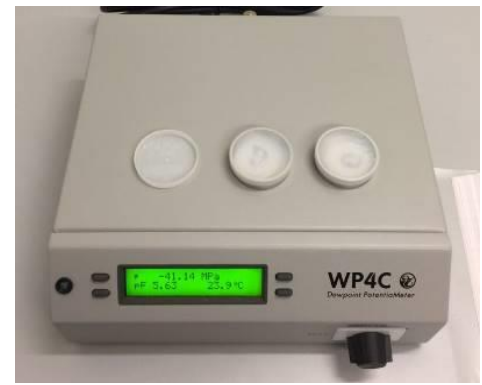

a. A photo

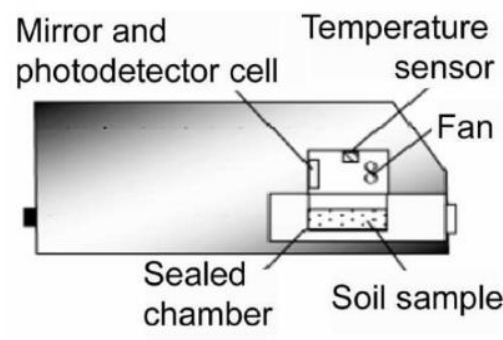

b. A schematic [49]

Fig.6 The chilled-mirror dew-point psychrometer (model: WP4C)

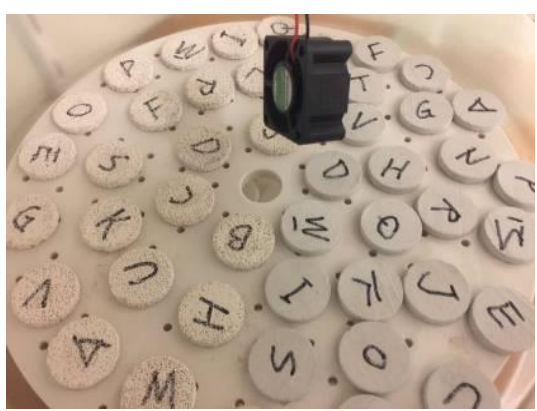

a. Samples under pre-conditioning

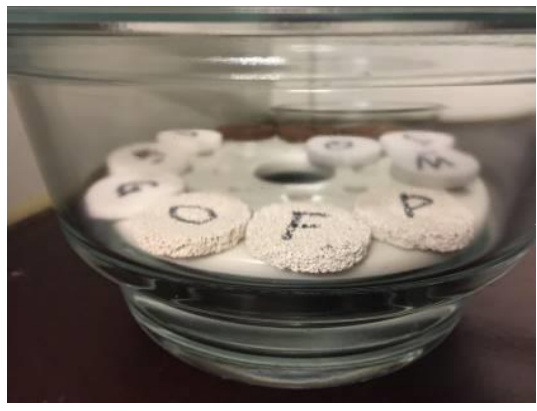

c. A close look of samples in the test

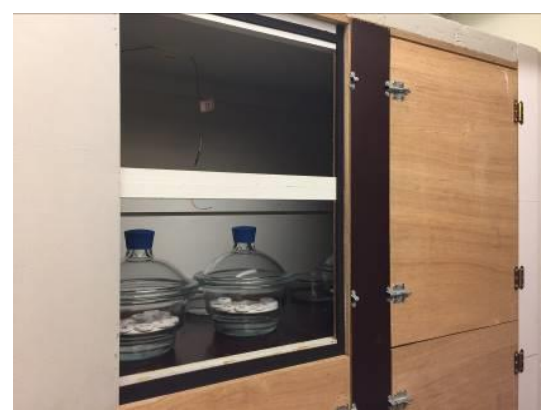

b. Samples in the insulation chamber

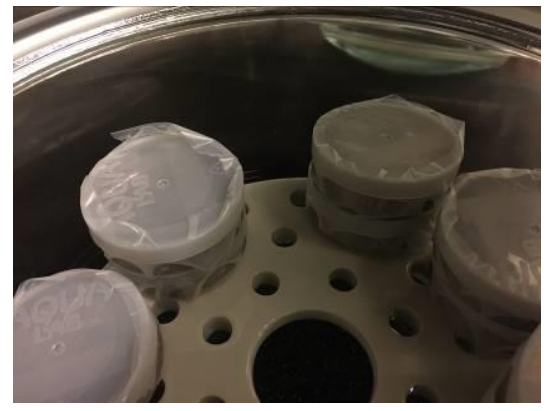

d. Samples sealed in cups

Fig.7 The psychrometer method for measuring moisture storage curves 
Postprint: Feng C, Janssen H. 2019. Hygric properties of porous building materials (IV): semi-permeable membrane and psychrometer methods for measuring moisture storage curves, Building and Environment, 152: 39-49.

doi: https://doi.org/10.1016/j.buildenv.2019.01.054

It should be noted that in the psychrometer test we always weigh the sample first and then measure its $p_{c}$, and the working principle of the chilled-mirror dew-point psychrometer subsequently makes an underestimation of the original sample $p_{c}$ (or an equivalent overestimation of $w$ ), inevitable due to the evaporation. However, the total volume of the sealed chamber can be estimated as $50 \mathrm{~mL}$. A simple calculation reveals that under our experimental conditions $1.1 \mathrm{mg}$ water vapor could yield $100 \%$ ambient RH in such a volume. Our sample size is roughly $3.5 \cdot 10^{-6} \mathrm{~m}^{3}$, corresponding to an overestimation of $w$ around $0.3 \mathrm{~kg} / \mathrm{m}^{3}$, which is completely negligible.

It should also be mentioned that the purpose for pre-conditioning samples at $97 \% \mathrm{RH}$ in the adsorption tests is to slow down the moisture absorption process when samples are exposed above pure water, so that the $p_{c}$ and $w$ profiles inside the sample are more uniform. For the same reason, the desorption tests are carried out at relatively high RHs. In our trial measurements, the $p_{c}$ evolutions of some samples are monitored after their sealing. It is observed that during the initial hours there are still some $p_{c}$ changes, which should be mainly attributed to the moisture content re-distribution within the sample. After several hours, equilibrium has almost been reached and the measured $p_{c}$ only shows minor fluctuations. For this reason, we always carry out the $p_{c}$ measurements on samples having been standing overnight.

\section{Results and discussion}

In this section, we will first report the results obtained through the semi-permeable membrane test and the psychrometer test. Next, the desorption results from both tests are confronted with each other, as well as with the outcomes of other tests. After that, the adsorption results obtained from these two methods are compared.

\subsection{Results from semi-permeable membrane and psychrometer tests}

Fig. 8 and 9 illustrate the experimental results obtained from the semi-permeable membrane test and the psychrometer test, respectively. For all three target materials both the adsorption and desorption curves are obtained. Several general remarks can be made to grasp a first interpretation of these results.

To start with, we can observe from both Fig. 8 and 9 that within the $p_{c}$ range lower than $-3.2 \cdot 10^{5} \mathrm{~Pa}$ $\left(\log _{10}\left(-p_{c}\right)>5.5\right)$, the desorption curves starting from $w_{s a t}$ and $w_{c a p}$ overlap with each other, whatever the material is. This interesting phenomenon indicates that for these materials the desorption hysteresis is not significant in this $p_{c}$ range, triggering inspiration for future studies.

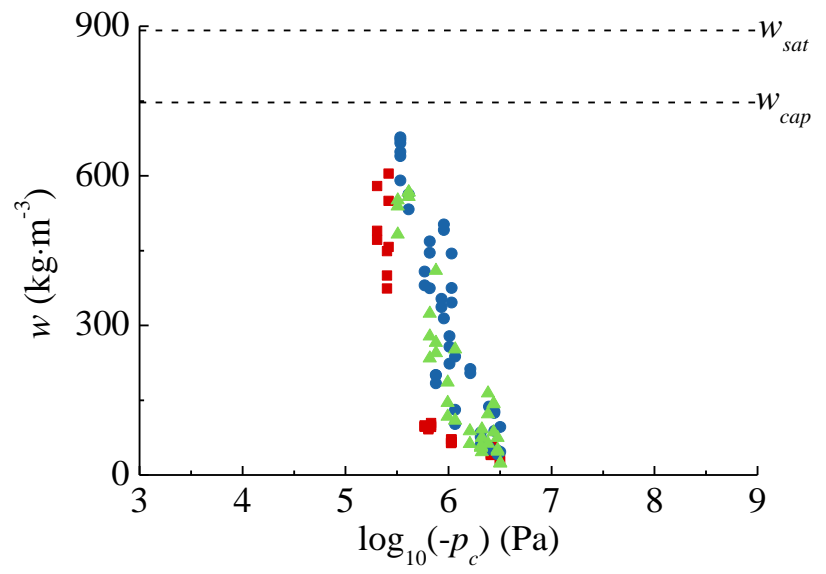

a. CS

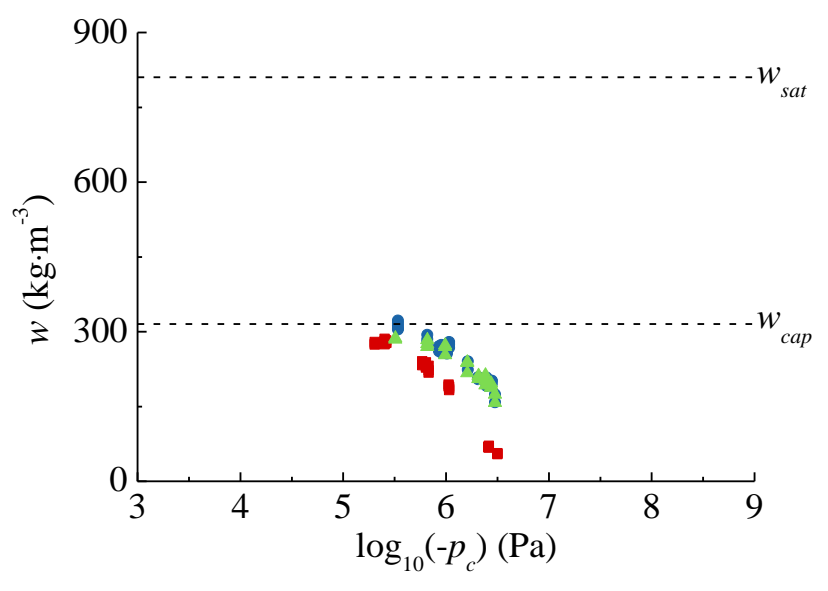

b. AAC 
Postprint: Feng C, Janssen H. 2019. Hygric properties of porous building materials (IV): semi-permeable membrane and psychrometer methods for measuring moisture storage curves, Building and Environment, 152: 39-49.

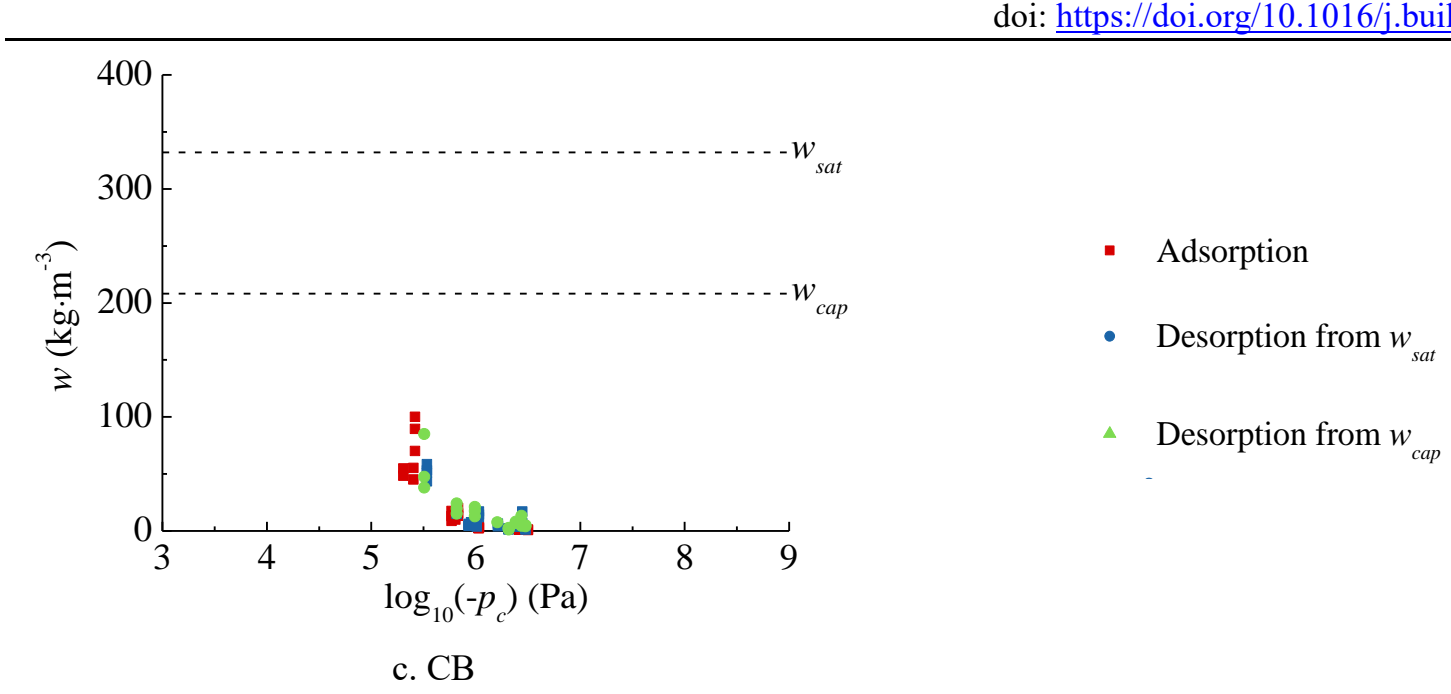

Fig.8 The moisture storage curves obtained from the semi-permeable membrane test

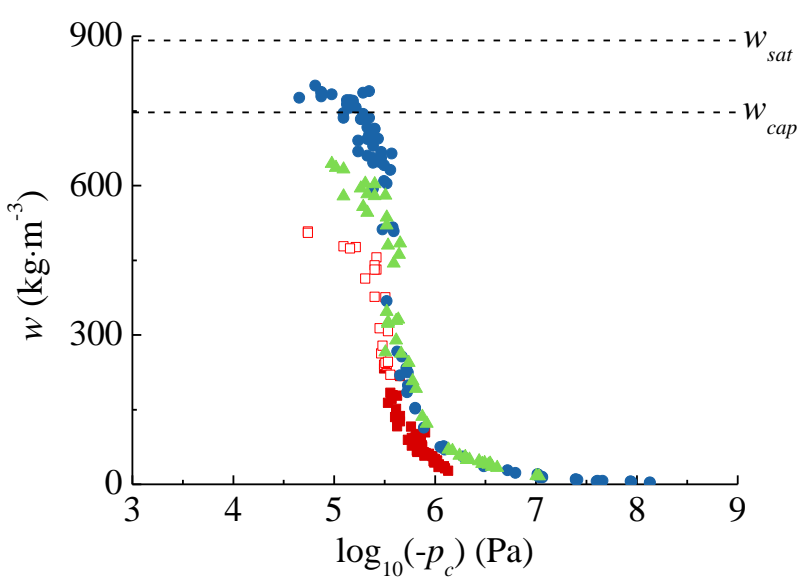

a. CS

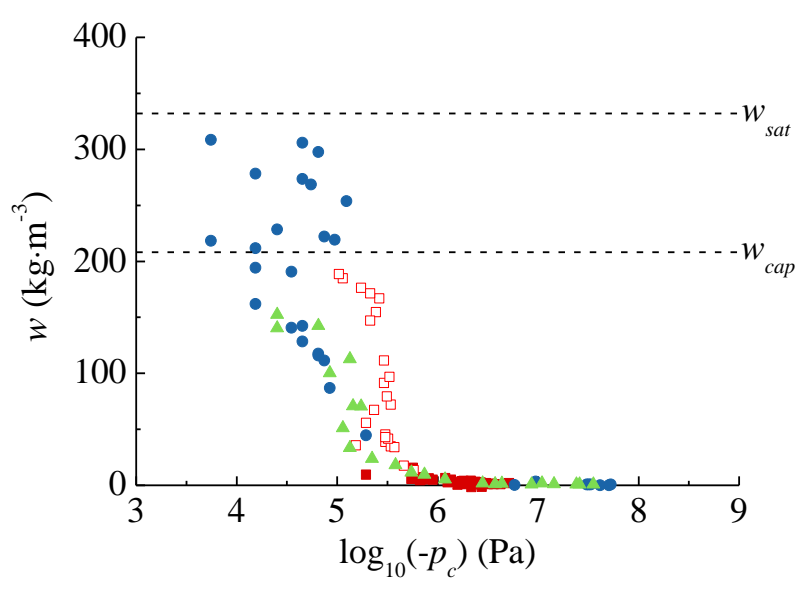

c. CB

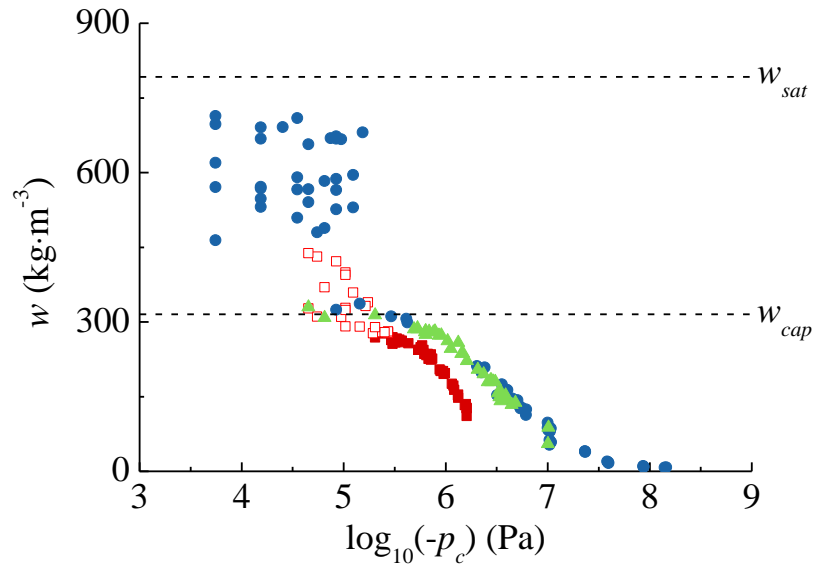

b. AAC
- Adsorption (vapor)

- Adsorption (water drop)

- Desorption from $w_{\text {sat }}$

Desorption from $w_{c a p}$

Fig.9 The moisture storage curves obtained from the psychrometer test

What's more, as is clearly revealed in Fig.8, we fail to obtain reliable results for capillary pressures above $-1 \cdot 10^{5} \mathrm{~Pa}\left(\log _{10}\left(-p_{c}\right)<5\right)$ with the semi-permeable membrane method. The main reason is that this extremely high humidity inevitably leads to severe condensation due to the temperature fluctuations beyond the control capability in our lab. 
Postprint: Feng C, Janssen H. 2019. Hygric properties of porous building materials (IV): semi-permeable membrane and psychrometer methods for measuring moisture storage curves, Building and Environment, 152: 39-49.

doi: https://doi.org/10.1016/j.buildenv.2019.01.054

In addition, the protocol of applying tiny water drops on the sample for accelerating the psychrometer adsorption process appears to yield reasonable results, which is in accordance with other studies [8]. Even with this protocol though, the adsorption measurement is still very slow: its complete execution takes more than 6 months. Consequently, for capillary pressures above $-1 \cdot 10^{5} \mathrm{~Pa}$, no adsorption curve results are collected with the psychrometer test, either.

Moreover, limited by the instrumental accuracy, large scatters of the data points are displayed for the desorption curves obtained from the psychrometer test for capillary pressures above $-1 \cdot 10^{5} \mathrm{~Pa}$. This is especially obvious for CB (Fig.9 c), who has relatively large pores, with a median radius around $4 \cdot 10^{-6} \mathrm{~m}$ (Table 2). Consequently, a large drop/rise of the storage curves around $-3.2 \cdot 10^{4} \mathrm{~Pa}\left(\log _{10}\left(-p_{c}\right)\right.$ $\approx 4.5)$ is expected, beyond the accuracy of our psychrometer $\left( \pm 5 \cdot 10^{4} \mathrm{~Pa}\right.$ in this range). Within this high humidity range the severe condensation incurred by temperature fluctuations also plays a role.

Last but not least, although all other 5 cases are reasonable, the hysteresis phenomenon of CB in the psychrometer test seems illogical (Fig.9 c), as the desorption curves should stay above the adsorption curve. At this moment we don't have a convincing explanation for this exception.

From the discussion above, we can build the first impression that both the semi-permeable membrane test and the psychrometer test work reasonably in the $p_{c}$ range below $-1 \cdot 10^{5} \mathrm{~Pa}$. Next, we will validate the experimental results.

\subsection{Comparison of results from different methods}

To validate the results obtained from the semi-permeable membrane test and the psychrometer test, we make a comparison between them on the results of all three materials. The pressure plate test and the desiccator test are used for external verification where applicable. Fig.10-12 illustrate these results.

Fig. 10 compares the desorption results obtained from different methods, all starting from $w_{\text {sat }}$. As is clearly reflected, the experimental results obtained from the semi-permeable membrane test and the psychrometer test agree with each nicely in the $p_{c}$ range below $-1 \cdot 10^{5} \mathrm{~Pa}$. Since these are two independent experimental methods (although the same psychrometer is used for $p_{c}$ determination) and provide very similar results, it is highly plausible that both methods are capable of delivering reliable results. Moreover, the pressure plate measurements provide almost overlapping results in the overhygroscopic range, while the results obtained from the desiccator test nicely extend to the results obtained from the semi-permeable membrane test and the psychrometer test. Since the pressure plate and the desiccator tests are well-established methods, their results convincingly support the correctness of the two novel methods.

Fig.11 illustrates the desorption results starting from $w_{\text {cap }}$. Similar to Fig.10, data points obtained from the semi-permeable membrane test, the psychrometer test and the pressure plate test agree with each other almost perfectly in the $p_{c}$ range below $-1 \cdot 10^{5} \mathrm{~Pa}$, confirming the reliability of all three methods. Although the desiccator results are not available as references in this case, the limited hysteresis of the target materials (discussed in Section 3.1) makes them redundant. We can therefore still assert that both the semi-permeable membrane method and the psychrometer method are trustworthy for the desorption measurement starting from $w_{\text {cap }}$.

Finally, Fig. 12 summarizes the adsorption results. Since by far there is no widely accepted protocol for measuring the adsorption storage curve in the over-hygroscopic range, we can only compare the results from the semi-permeable membrane method and the psychrometer method. Fortunately, these two novel methods produce very similar results in the over-hygroscopic range, with the desiccator test as an optimistic external support from the hygroscopic range (for CS and AAC only). Based on this, as well as the success of the semi-permeable membrane method and the psychrometer method for the 
Postprint: Feng C, Janssen H. 2019. Hygric properties of porous building materials (IV): semi-permeable membrane and psychrometer methods for measuring moisture storage curves, Building and Environment, 152: 39-49.

doi: https://doi.org/10.1016/j.buildenv.2019.01.054

desorption processes, it is reasonable to remain confident for their adsorption results. However, the strange hysteresis phenomenon of CB in the psychrometer test (Fig.9 c) calls for further study of its adsorption test, which remains as future work.

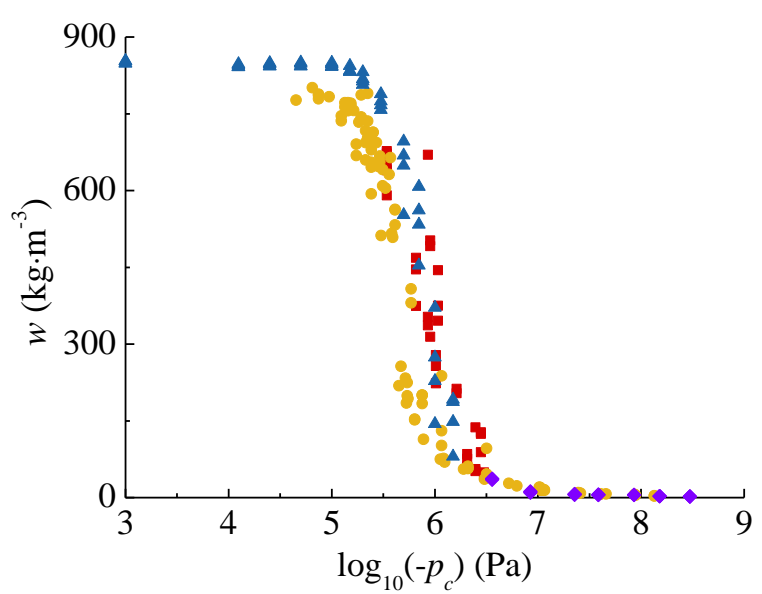

a. CS

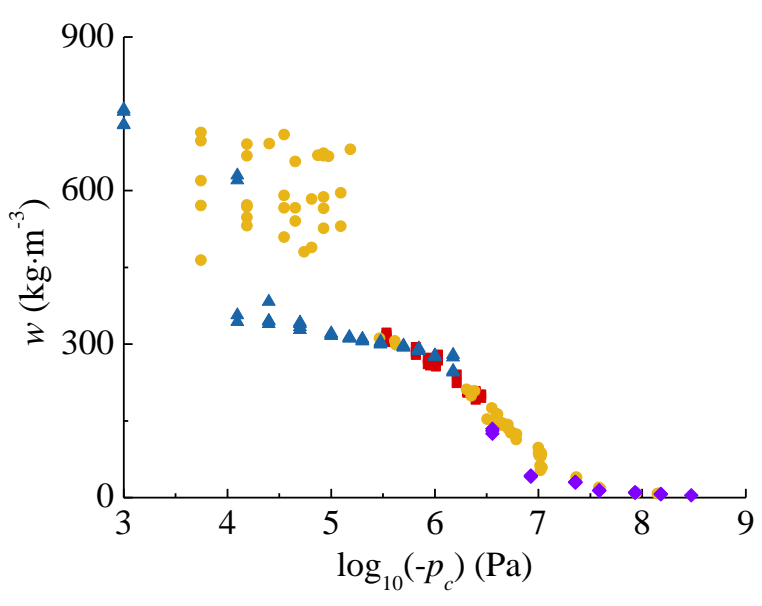

b. AAC

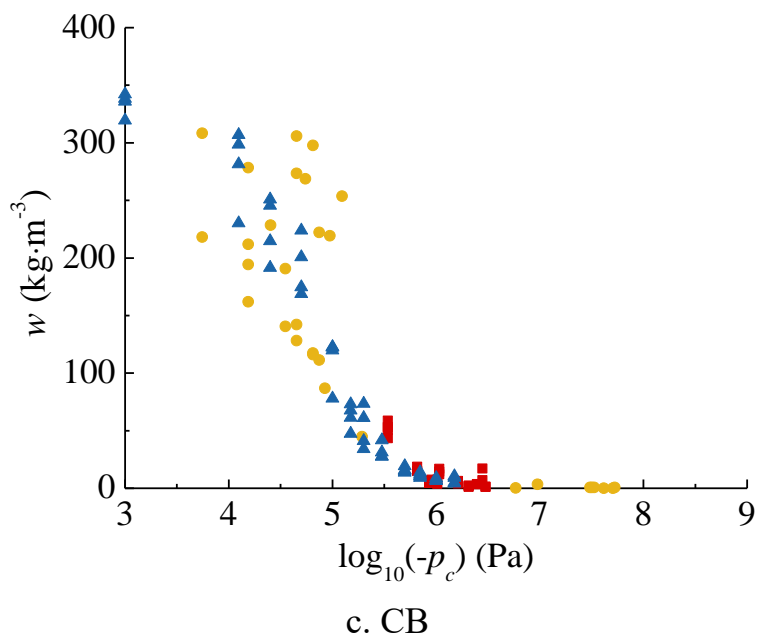

- Semi-permeable membrane

- Psychrometer

- Pressure plate

- Desiccator

Fig.10 Comparison of the desorption results from different tests (starting from $w_{\text {sat }}$ )

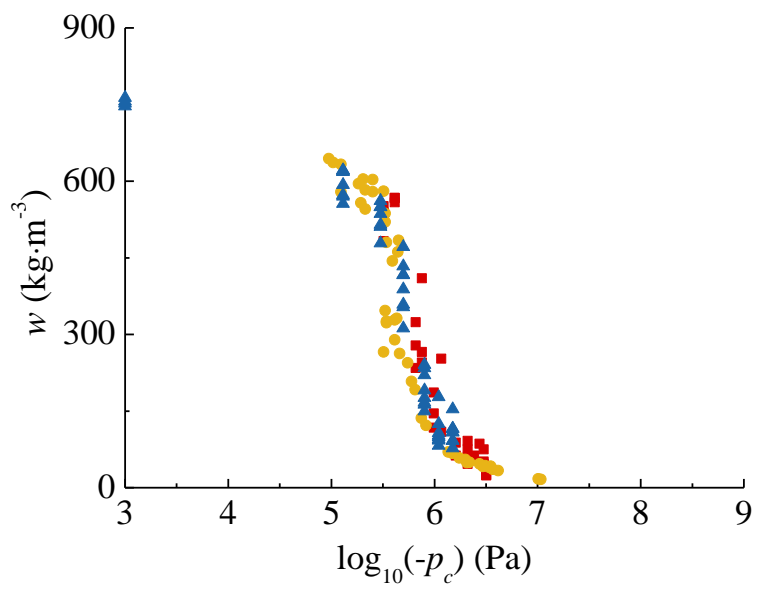

a. CS

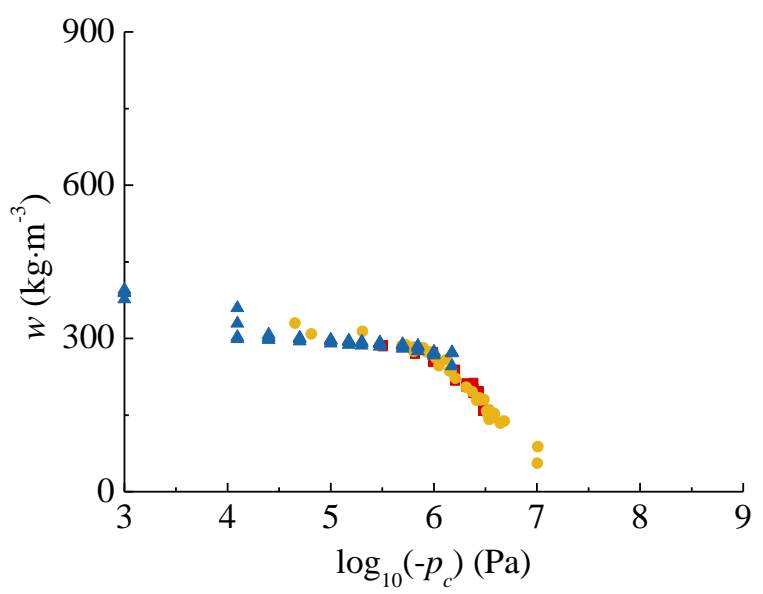

b. AAC 

psychrometer methods for measuring moisture storage curves, Building and Environment, 152: 39-49.

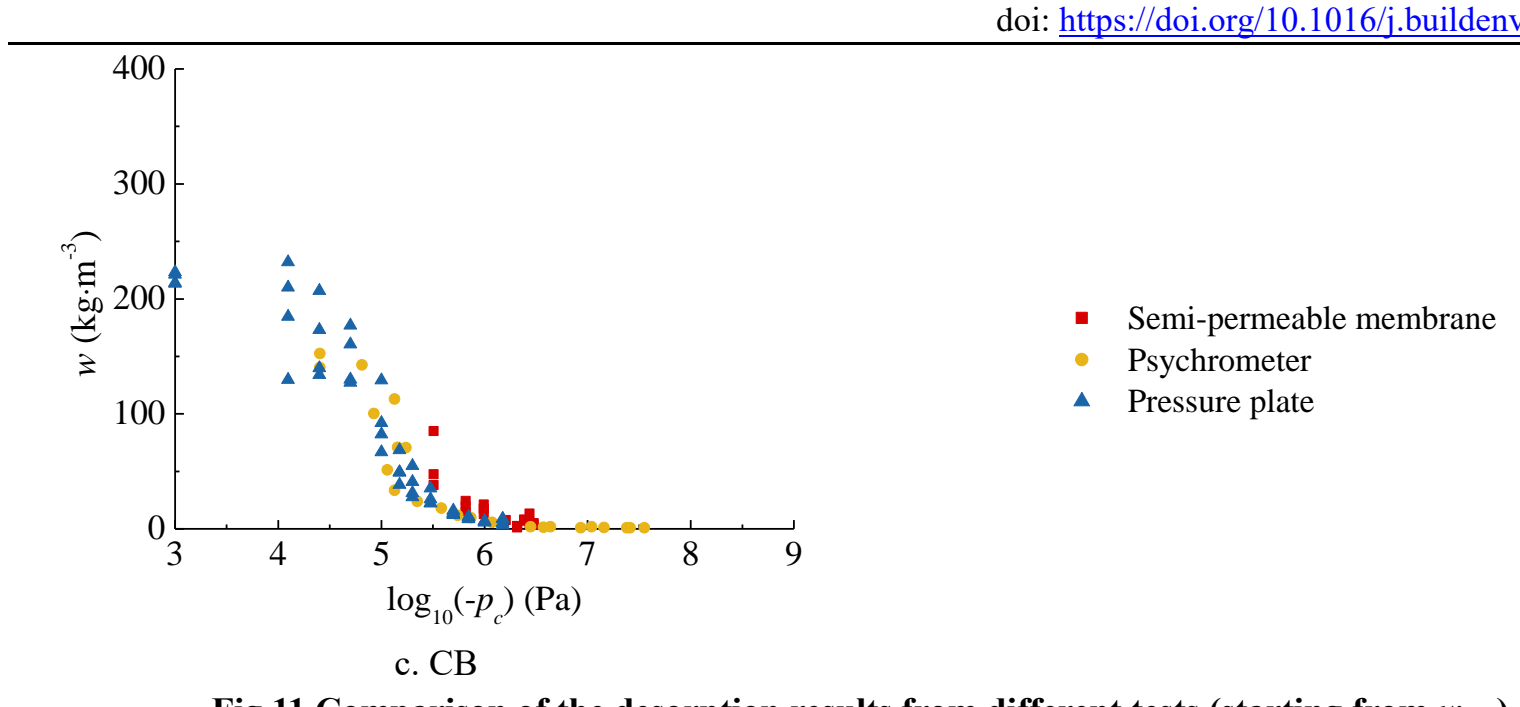

Fig.11 Comparison of the desorption results from different tests (starting from $w_{c a p}$ )

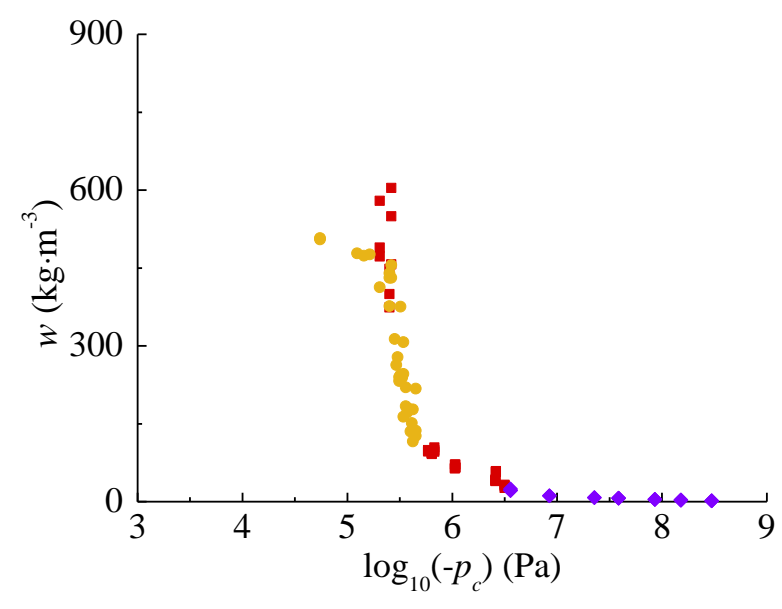

a. CS

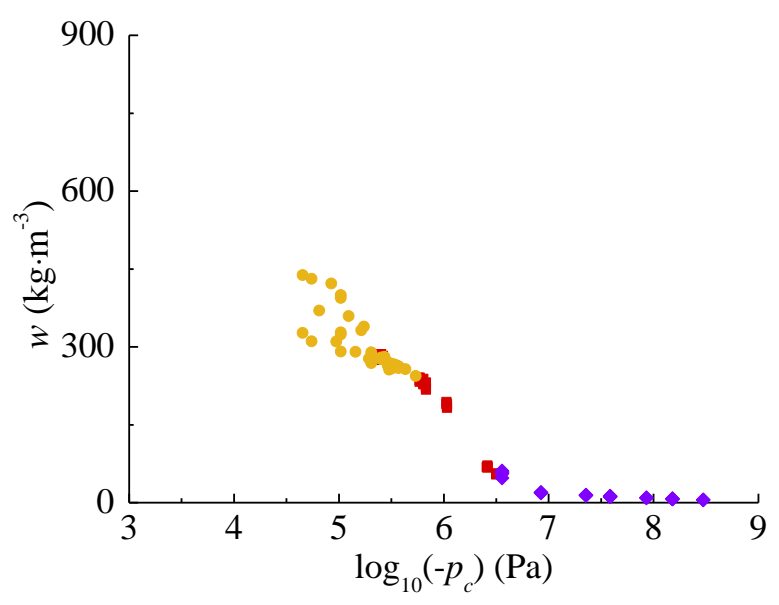

b. AAC

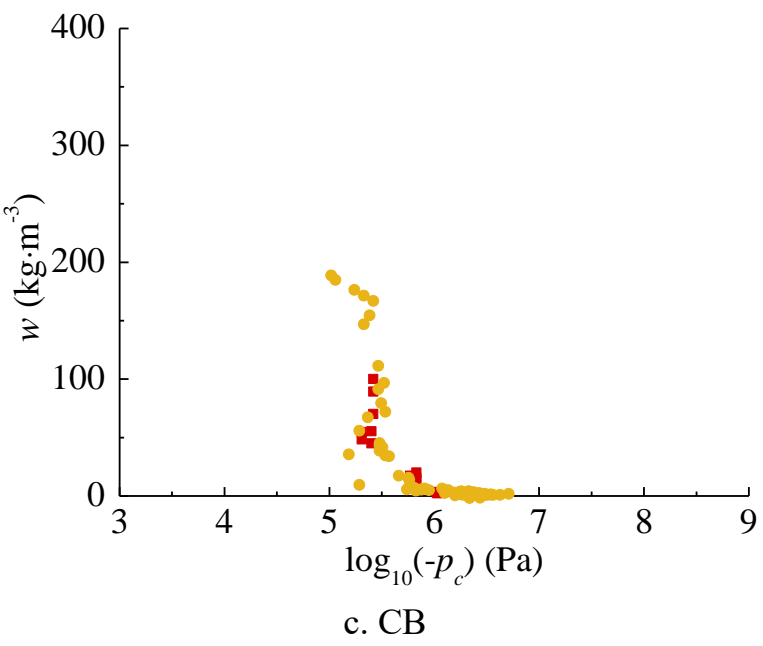

- Semi-permeable membrane

- Psychrometer

- Desiccator

Fig.12 Comparison of the adsorption results from different tests

For more quantitative evaluations, we fit the data points obtained from the semi-permeable membrane method, the psychrometer method and the pressure plate method into respective moisture storage curves. The adjusted $R^{2}$ from the fittings directly reflects whether a method produces accurate results or not. Moreover, to evaluate the closeness of data obtained from these three methods, we lump all the data together, repeat the fitting and check the adjusted $R^{2}$ again. Since both desorption and 
adsorption share the same experimental principle, we focus on the desorption process from $w_{\text {sat }}$ only.

For the fittings, the well-known and widely accepted van Genuchten model [50] is adopted:

$$
w=w_{0} \cdot \sum_{i=1}^{k} l_{i}\left[1+\left(a_{i} \cdot p_{c}\right)^{b_{i}}\right]^{\frac{1-b_{i}}{b_{i}}}
$$

where $w_{0}, a_{i}$ and $b_{i}$ are all fitting parameters, while $l_{i}$ is the weighing factor for system $i$ with the constraint $\sum_{i=1}^{k} l_{i}=1$. The fitting parameter $w_{0}$ is fixed as $w_{\text {sat }}$ in our case. Trials show that for $i=1$ or 2 the fitting results are already very excellent, while additional systems don't provide noticeable improvement. Consequently, we keep $i=1$ or 2 in all our fittings.

As mentioned before, the semi-permeable membrane method and the psychrometer method are mainly applicable in the $p_{c}$ range below $-1 \cdot 10^{5} \mathrm{~Pa}$. Thus, for these two methods we only select data in this range for the fittings. Moreover, while conducting the lumped fittings, a total weighing factor of $1 / 3$ is assigned to each method, further averaged by the number of points obtained from that method.

Fig.13 illustrates the respective and lumped fittings for CS as an example, while Fig.14 compares the adjusted $R^{2}$ for all fittings. Clearly, the semi-permeable membrane method, the psychrometer method and the pressure plate method have similar adjusted $R^{2}$ values, and no method shows a dominant advantage. The adjusted $R^{2}$ values for the lumped fittings are also at the same level as for the respective fittings. This indicates that there are not significant and systematic deviations between different methods, otherwise the adjusted $R^{2}$ should have decrease obviously.

Based on the analysis above, we conclude that both the semi-permeable membrane method and the psychrometer method are reliable in obtaining the moisture storage curves of porous building materials in the over-hygroscopic range.

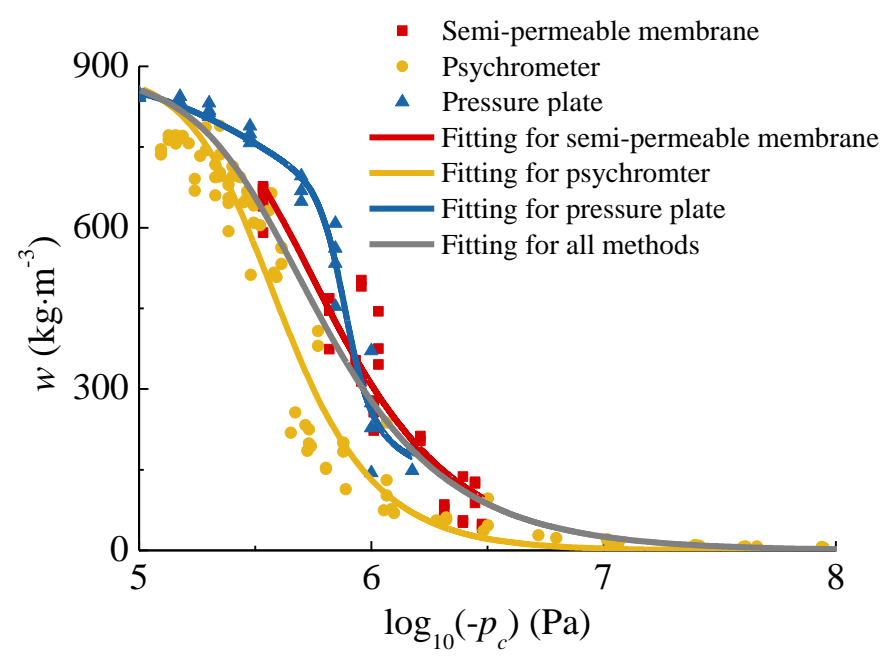

Fig.13 Fittings for the moisture storage curves of CS (desorption from $w_{\text {sat }}$ )

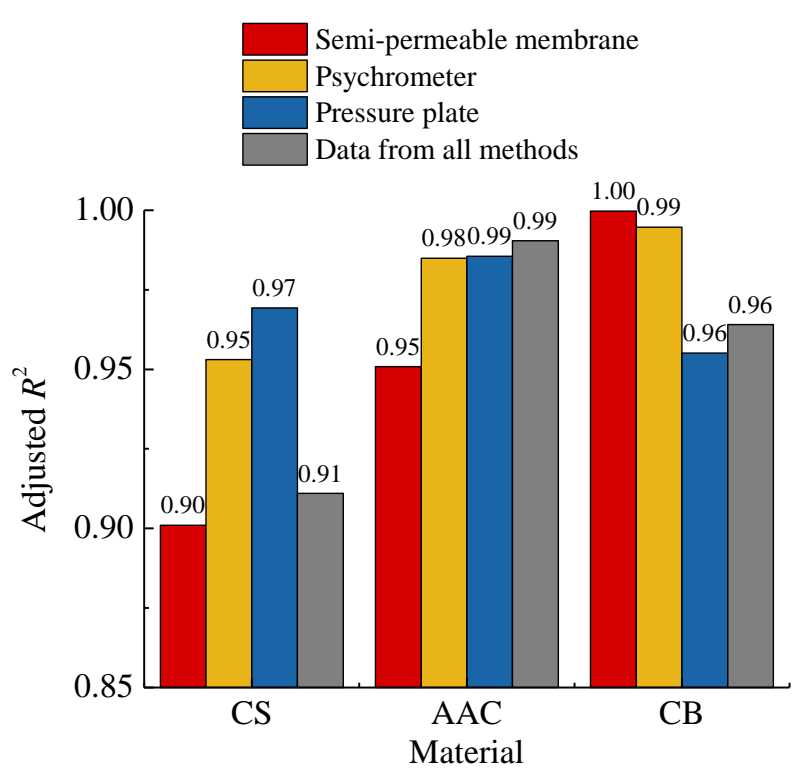

Fig.14 Comparison of the adjusted $R^{2}$ for fittings (desorption from $w_{\text {sat }}$ )

\subsection{Discussion}

Above we have validated the semi-permeable membrane method and the psychrometer method. To have a thorough understanding of their characteristics, some further remarks are needed.

The most attractive advantage of these two novel methods is their capability of measuring the 
Postprint: Feng C, Janssen H. 2019. Hygric properties of porous building materials (IV): semi-permeable membrane and psychrometer methods for measuring moisture storage curves, Building and Environment, 152: 39-49.

doi: https://doi.org/10.1016/j.buildenv.2019.01.054

adsorption curve (although slightly dubious still on CB). As explained in Section 1, the adsorption measurement on porous building materials in the over-hygroscopic range has been a difficulty for a long time. Although much effort has been made by different researchers and some methods are suggested - such as the modified pressure plate in Lund University [33] - there is no ideal and widely adopted solution. As two novel methods with great potential, both the semi-permeable membrane method and the psychrometer method resolve this problem (at least partially), providing a direct complement to current desorption experimental protocols, and hence making our measurements on the moisture storage curve in the over-hygroscopic range complete.

The next superiority of the semi-permeable membrane method and the psychrometer method is that they don't require expensive set-ups, and their experimental procedures are very simple. Both the semipermeable membrane and the psychrometer are commercially available in the market at a low price, with a lot of options to choose according to different requirements. The handling is just slightly more complicated than the desiccator method - the most popular method for measuring the moisture storage curves in the hygroscopic range - but is still much easier than the pressure plate and many other tests.

A third benefit of the semi-permeable membrane method and the psychrometer method is their efficiency. For the psychrometer method we admit that it is extremely slow for the adsorption measurement. However, for the desorption measurement 1 month already suffices, consuming the same time as most of the experimental methods listed in Table 1. When it comes to the semi-permeable membrane method, roughly 3 4 weeks are enough for both the adsorption and desorption measurements.

A final strong point is the applicable range of these two methods. In this study we just perform the measurements in the over-hygroscopic range, but the scope is already wider than the combination of the pressure plate method and the pressure membrane method $\left(0 \sim-1.0 \cdot 10^{7} \mathrm{~Pa}\right)$. Moreover, according to the working principles of the semi-permeable membrane method and the psychrometer method, the lower bound of their applicable range can obviously extend to the hygroscopic range. For the upper bound, both methods are not accurate when the $p_{c}$ is greater than $-1.0 \cdot 10^{5} \mathrm{~Pa}$ (and hence not applicable to $\mathrm{CB}$ or other materials with large pores), and some other method - such as the hanging water column [34] - should be employed.

To sum up, both the semi-permeable membrane method and the psychrometer method are satisfactory in measuring the moisture storage curves of porous building materials in the overhygroscopic range. They are cheap, simple, efficient and widely applicable. For the semi-permeable membrane method, the advantages are especially conspicuous. It is therefore advisable to standardized it as the over-hygroscopic part of the desiccator test, replacing the time-consuming and troublesome pressure plate/membrane test.

\section{Conclusions}

This paper proposes two novel methods - the semi-permeable membrane method and the psychrometer method - to determine the moisture storage curves of porous building materials in the over-hygroscopic range. Measurements on calcium silicate, autoclaved aerated concrete and ceramic brick are conducted and the results are validated by comparing the experimental results obtained from these two methods, as well as from other existing protocols. It has been proved that both the semipermeable membrane method and the psychrometer method can provide reliable results up to a capillary pressure of around $-1 \cdot 10^{5} \mathrm{~Pa}$ for both adsorption and desorption processes. Their other advantages - low cost, simple procedure, test efficiency and wide applicable range - make them very 
Postprint: Feng C, Janssen H. 2019. Hygric properties of porous building materials (IV): semi-permeable membrane and psychrometer methods for measuring moisture storage curves, Building and Environment, 152: 39-49.

advisable.

doi: https://doi.org/10.1016/j.buildenv.2019.01.054

\section{Acknowledgements}

This project is supported by the FWO Odysseus grant "Moisture transfer in building materials: analysis at the porescale level" (No. G.0C55.13N). We express sincere gratitude to Prof. Rafaela Cardoso in Instituto Superior Técnico for sharing her experience with the psychrometer, and to Prof. Bart Van der Bruggen in KU Leuven for sharing his expertise about semi-permeable membranes.

\section{References}

[1] van Aarle M, Schellen H, van Schijndel J. Hygro Thermal Simulation to Predict the Risk of Frost Damage in Masonry; Effects of Climate Change. Energy Procedia. 2015;78:2536-41.

[2] Harrestrup M, Svendsen S. Full-scale test of an old heritage multi-storey building undergoing energy retrofitting with focus on internal insulation and moisture. Building and Environment. 2015;85:123-33.

[3] Woloszyn M, Kalamees T, Olivier Abadie M, Steeman M, Sasic Kalagasidis A. The effect of combining a relative-humidity-sensitive ventilation system with the moisture-buffering capacity of materials on indoor climate and energy efficiency of buildings. Building and Environment. 2009;44:515-24.

[4] Fang L, Clausen G, Fanger PO. Impact of temperature and humidity on the perception of indoor air quality. Indoor Air. 1998;8:8090

[5] Funk M, Wakili KG. Driving potentials of heat and mass transport in porous building materials: a comparison between general linear, thermodynamic and micromechanical derivation schemes. Transport in Porous Media. 2008;72:273-94.

[6] Hens H, Ojanen T, Künzel HM, Dow G, Rode C, Hagentoft C. IEA Annex 24: Heat, Air and Moisture Transfer in Insulated Envelope Parts. Final Report, Volume 1, Task 1: Modeling. 1996.

[7] Woloszyn M, Rode C. IEA Annex 41: Whole Building Heat, Air, Moisture Response. Subtask 1: Modeling Principles and Common Exercises. 2008.

[8] Lu Y, Abuel-Naga H, Bouazza A. Water retention curve of GCLs using a modified sample holder in a chilled-mirror dew-point device. Geotextiles and Geomembranes. 2017;45:23-8.

[9] Hilf JW. An investigation of pore-water pressure in compacted cohesive soils. US Department of Interior Bureau of Reclamation, Technical Memorandum 654, Denver, Colorado. 1956.

[10] Mavroulidou M, Zhang X, Cabarkapa Z, Gunn M. A study of the laboratory measurement of the Soil Water Retention Curve. 11 th International Conference on the Environmental Science and Technology. Chania, Crete, Greece 2009. A 907-15.

[11] Southen JM, Kerry Rowe R. Evaluation of the water retention curve for geosynthetic clay liners. Geotextiles and Geomembranes. 2007;25:2-9.

[12] Tarantino A, Romero E, Cui YJ. Laboratory and field testing of unsaturated soils: Springer; 2009.

[13] Abuel-Naga H, Bouazza A. A novel laboratory technique to determine the water retention curve of geosynthetic clay liners. Geosynthetics International. 2010;17:313-22.

[14] Bannour H, Stoltz G, Delage P, Touze-Foltz N. Effect of stress on water retention of needlepunched geosynthetic clay liners. Geotextiles and Geomembranes. 2014;42:629-40.

[15] Delage P, Suraj de Silva GPR, Vicol T. Suction controlled testing of non saturated soils with an osmotic consolidometer. Proceedings 7th International Conference on Expansive Soils. Dallas. 1992:206-11.

[16] Feng C, Janssen H, Wu C, Feng Y, Meng Q. Validating various measures to accelerate the static gravimetric sorption isotherm determination. Building and Environment. 2013;69:64-71.

[17] Garbalińska H, Bochenek M, Malorny W, von Werder J. Comparative analysis of the dynamic vapor sorption (DVS) technique and the traditional method for sorption isotherms determination - Exemplified at autoclaved aerated concrete samples of four density classes. Cement and Concrete Research. 2017;91:97-105.

[18] Delage P, Mantikos V, Ackerley S, Kirkham A, Tsiampousi A, Taborda DMG, et al. Investigating soil water retention characteristics 
Postprint: Feng C, Janssen H. 2019. Hygric properties of porous building materials (IV): semi-permeable membrane and psychrometer methods for measuring moisture storage curves, Building and Environment, 152: 39-49.

doi: https://doi.org/10.1016/j.buildenv.2019.01.054

at high suctions using relative humidity control. E3S Web of Conferences. 2016;9:10007.

[19] Vangpaisal T, Bouazza A. Gas Permeability of Partially Hydrated Geosynthetic Clay Liners. Journal of Geotechnical and Geoenvironmental Engineering. 2004;130:93-102.

[20] Bouazza A, Vangpaisal T. An apparatus to measure gas permeability of geosynthetic clay liners. Geotextiles and Geomembranes. 2003;21:85-101.

[21] Beddoe RA, Take WA, Rowe RK. Water-Retention Behavior of Geosynthetic Clay Liners. Journal of Geotechnical and Geoenvironmental Engineering. 2011;137:1028-38.

[22] Madsen HB, Jensen CR, Boysen T. A comparison of the thermocouple psychrometer and the pressure plate methods for determination of soil water characteristic curves. Journal of Soil Science. 1986;37:357-62.

[23] Acikel AS, Singh RM, Bouazza A, Gates WP, Rowe RK. Applicability and accuracy of the initially dry and initially wet contact filter paper tests for matric suction measurement of geosynthetic clay liners. Géotechnique. 2015;65:780-7.

[24] ASTM D5298 - 16: Standard Test Method for Measurement of Soil Potential (Suction) Using Filter Paper. 2016.

[25] Roels S, Elsen J, Carmeliet J, Hens H. Characterisation of pore structure by combining mercury porosimetry and micrography. Materials and structures. 2001;34:76-82.

[26] ISO 12571: 2013(E) Hygrothermal performance of building materials and products - Determination of hygroscopic sorption properties. 2013.

[27] ASTM C1498 - 2010: Standard Test Method for Hygroscopic Sorption Isotherms of Building Materials. 2010.

[28] ASTM C1699 - 09: Standard Test Method for Moisture Retention Curves of Porous Building Materials Using Pressure Plates. 2009.

[29] ISO 11274: 1998(E) Soil quality - Determination of the water retention characteristic - Laboratory methods. 1998.

[30] ASTM D4404 - 18: Standard Test Method for Determination of Pore Volume and Pore Volume Distribution of Soil and Rock by Mercury Intrusion Porosimetry. 2018.

[31] Feng C, Janssen H, Feng Y, Meng Q. Hygric properties of porous building materials: Analysis of measurement repeatability and reproducibility. Building and Environment. 2015;85:160-72.

[32] Hansen MH. Retention curves measured using pressure plate and pressure membrane apparatus: Description of method and interlaboratory comparison. Nordtest Technical Report 367. 1998.

[33] Fredriksson M, Johansson P. A Method for Determination of Absorption Isotherms at High Relative Humidity Levels: Measurements on Lime-Silica Brick and Norway Spruce (Picea abies(L.) Karst.). Drying Technology. 2016;34:132-41.

[34] Plagge R, Scheffler G, Nicolai A. Experimental methods to derive hygrothermal material functions for numerical simulation tools. Building X Conference. Clearwater, Florida 2007. 1-12.

[35] Bittelli M, Flury M. Errors in water retention curves determined with pressure plates. Soil Science Society of America Journal. 2009;73:1453-60.

[36] Day RL, Marsh BK. Measurement of porosity in blended cement pastes. Cement and Concrete Research. 1988;18:63-73.

[37] Diamond S. Mercury porosimetry: an inappropriate method for the measurement of pore size distributions in cement-based materials. Cement and Concrete Research. 2000;30:1517-25.

[38] Feng C, Janssen H. Hygric properties of porous building materials (III): Impact factors and data processing methods of the capillary absorption test. Building and Environment. 2018;134:21-34.

[39] Safiuddin M, Hearn N. Comparison of ASTM saturation techniques for measuring the permeable porosity of concrete. Cement and Concrete Research. 2005;35:1008-13.

[40] ISO 12572: 2001(E) Hygrothermal performance of building materials and products - Determination of water vapour transmission properties. 2001.

[41] Gao Z, Hu Q. Estimating permeability using median pore-throat radius obtained from mercury intrusion porosimetry. Journal of Geophysics and Engineering. 2013;10:025014.

[42] Feng C, Janssen H. Hygric properties of porous building materials (II): Analysis of temperature influence. Building and Environment. 2016;99:107-18. 
Postprint: Feng C, Janssen H. 2019. Hygric properties of porous building materials (IV): semi-permeable membrane and psychrometer methods for measuring moisture storage curves, Building and Environment, 152: 39-49.

doi: https://doi.org/10.1016/j.buildenv.2019.01.054

[43] Roels S, Carmeliet J, Hens H, Adan O, Brocken H, Czerny R, et al. HAMSTAD Work Package 1: Final Report - Moisture Transfer Properties and Materials Characterisation, EU Contract GRD1-1999-20007. 2003.

[44] Richards L. Porous plate apparatus for measuring moisture retention and transmission by soil. Soil Science. 1948;66:105-9.

[45] Cresswell H, Green T, McKenzie N. The adequacy of pressure plate apparatus for determining soil water retention. Soil Science Society of America Journal. 2008;72:41-9.

[46] https://www.lenntech.com/Data-sheets/Filmtec-Flat-Sheet-Membrane-Chart-L.pdf.

[47] Cardoso R, Romero E, Lima A, Ferrari A. A comparative study of soil suction measurement using two different high-range psychrometers. Experimental unsaturated soil mechanics: Springer; 2007. p. 79-93.

[48] https://www.metergroup.com/environment/products/wp4c/.

[49] Leong E-C, Tripathy S, Rahardjo H. Total suction measurement of unsaturated soils with a device using the chilled-mirror dewpoint technique. Géotechnique. 2003;53:173-82.

[50] van Genuchten MT. A closed-form equation for predicting the hydraulic conductivity of unsaturated soils. Soil Science Society of America Journal. 1980;44:892-8. 